(C) 2010 IEEE. Personal use of this material is permitted. Permission from IEEE must be obtained for all other uses, in any current or future media, including reprinting/republishing this material for advertising or promotional purposes, creating new collective works, for resale or redistribution to servers or lists, or reuse of any copyrighted component of this work in other works. 


\title{
Iterative Automatic Segmentation in Cardiac PET Based on TAC Correlation: Preliminary Results
}

\author{
José M. Mateos-Pérez, Student Member, IEEE, Carmen García-Villalba, Michael Dae, Mónica Abella, Member, \\ IEEE, Manuel Desco, and Juan José Vaquero, Senior Member, IEEE
}

\begin{abstract}
Conventional kinetic parameter estimation based on compartmental models requires an accurate estimation of arterial blood input function. To avoid invasive blood sampling, an image-derived input function can be obtained by manually defining a Region of Interest. Here we propose a new and simple, iterative method for automatic segmentation and input function calculation of PET cardiac studies using correlation as a distance metric between a priori information regarding the approximate shape of the final time-activity curve (TAC) and the actual TAC extracted from the image temporal series.
\end{abstract}

\section{INTRODUCTION}

$\mathrm{Q}$ UANTITATIVE kinetic analysis of dynamic cardiac PET data provides unique information that can enable improved discrimination between different states of the myocardium tissue. Conventional kinetic parameter estimation based on compartmental models requires an accurate estimation of arterial blood input function (IF). To avoid invasive blood sampling on those applications where it can be challenging, an image-derived IF (IDIF) can be obtained by manually defining a Region Of Interest (ROI) [1-4]. Manual segmentation of these images may have reliability and reproducibility problems and is also a time-consuming process; therefore, several automatic time activity-curve (TAC) extraction techniques have been developed; usually, image segmentation is a byproduct of this process. Factor analysis of dynamic structures (FADS) is currently the most used tool to perform automatic segmentations [5, 6], but its main problem, the non-uniqueness of the solution, implies spatial overlapping between the resulting factor images; this also means that a certain region TAC will spill into others. Several approaches have been tried to solve this $[7,8]$ by imposing additional restrictions on the values of the factors used. Several other approaches have been proposed in the literature involving covariance maps, clustering and

Manuscript received November 12, 2010. This work was supported in part by the CENIT-AMIT Ingenio 2010, Ministerio de Ciencia e Innovación, TEC2007-64731, TEC 2008-06715-C02-1, RETIC-RECAVA, Ministerio de Sanidad y Consumo, and the ARTEMIS de la Comunidad de Madrid (S2009/DPI-1802) programs.

José María Mateos-Pérez, Carmen García-Villalba and Mónica Abella are with Unidad de Medicina y Cirugía Experimental, Hospital General Universitario Gregorio Marañón, Madrid, Spain (e-mail: jmmateos@mce.hggm.es, cgarcia@mce.hggm.es, mabella@mce.hggm.es. Telephone: +34 91 4265067).

Michael Dae is with University of California, San Francisco, USA.

Manuel Desco and Juan José Vaquero are with the Departamento de Bioingeniería e Ingeniería Aeroespacial, Universidad Carlos III de Madrid, Spain (e-mail: manuel.desco@uc3m.es, juanjose.vaquero@uc3m es). independent component analysis (ICA) [9-11]. However, they still suffer from spatial overlapping, have not been developed further or results for this particular application have not been published.

In order for these tools to become clear candidates for clinical use, they should comply with at least two requirements: 1) they have to be easy to use, and 2) they should provide consistent and reliable results.

Here we propose a new and simpler iterative method for automatic image-derived input function calculation of PET cardiac studies using a priori information with the main purpose of avoid spatial overlapping.

\section{MATERIAL AND MEthodS}

Three swine cardiac ${ }^{13} \mathrm{NH}_{3}$ dynamic PET studies were used in this preliminary study. $740 \mathrm{MBq}$ of ${ }^{13} \mathrm{NH}_{3}$ were injected in a bolus. Image matrix size was $128 \times 128 \times 47$, and voxel size was $2.34 \mathrm{~mm}$ x $2.34 \mathrm{~mm} \times 3.27 \mathrm{~mm}$. The 25 frames dynamic sequence was acquired for a total of 900 seconds ( $18 \times 5$ s, 2 x 15s, 3 x 60s, 2 x 300 s). Three different ROIs were drawn by a specialist over one randomly chosen study in order to segment left ventricle, right ventricle and myocardium. From those three ROIs, three TACs, one for each region, were extracted. The right and left ventricle TACs were fitted to the following gamma function:

$$
f(t)=K t^{\alpha} e^{-\frac{t}{\beta}}
$$

The myocardium curve was modelled after an exponential plus a constant factor that corrects for the late time plateau:

$$
f(t)=K\left(1-e^{-\frac{t}{\alpha}}\right)+\beta t
$$

Exact values of $K, \alpha$, and $\beta$ in (1) and (2) depend on the particular set of curves used to obtain the template, but the overall shape is always the same: the left ventricle TAC is similar to the right ventricle one, with a delayed maximum and a slower decay, whereas the myocardium reaches a smoothly ascendant plateau (Fig. 1).

After the initial template curves generation, the method iteratively seeks the TACs corresponding to the three regions using the templates as an initial estimation of the solution. Then, in every step of the iteration, correlation maps are 
calculated. A correlation map is a static image with the same spatial dimensions as the volume being analyzed; the value for each voxel in the map is the correlation between the TAC in the original study and the template. Therefore, three correlation images are generated as a result.

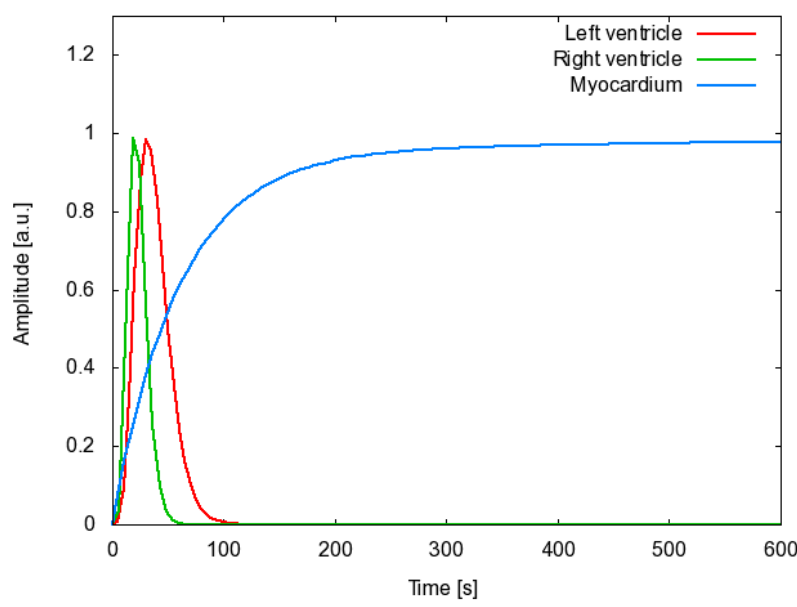

Fig. 1. Sample curves used to start the iterations. The left and right ventricle curves originate from (1) $(K=3.045 \mathrm{e}-6, \alpha=5.2, \beta=6 \mathrm{for}$ left ventricle; $K=1$ 15e-6, $\alpha=6.8, \beta=3$ for right ventricle) and the myocardium curve originates from (2) $(K=0.956, \alpha=59.71, \beta=3.85 \mathrm{e}-5)$. The amplitudes for each curve (controlled by constant $K$ ) have been set so that each maximum approaches 1 .

There are three main operations on each iteration. The first one is carried out for every voxel in the dynamic volume and the last two involve the correlation images generated at the first step:

1. The correlation between the measured TAC for each voxel and the three templates in the model is computed. The highest correlation value is assigned to the corresponding ROI correlation map if it is higher than a predefined threshold (currently, a correlation coefficient of $\rho=0.5$ is used for the ventricle curves and $\rho=0.55$ for the myocardium). This variable is a measurement of how much every curve can change its shape between iterations. At higher values, less amount of change is allowed. The other two correlation maps are set to zero for that voxel.

2. A mask is computed from each ROI correlation map using a median filter ( $3 \times 3$ pixels) to eliminate impulsive noise followed by thresholding: those voxels lower than one third of the maximum value for that slice are set to zero.

3. A new curve for each ROI is calculated by averaging the measured TACs of those voxels determined by the masks.

After every step, if less than 5\% of the voxels in the image have changed, the correlation threshold used in step 1 is incremented by a factor of $2 \%$. This is done to eliminate noisy voxels.

Convergence of this algorithm is studied by computing the sum of absolute differences between a TAC and the one obtained in the next iteration (Fig. 2).

9 iterations have been used in the tests presented in this work. The result consists of three correlation images (one for every template used) and three TACs extracted from the original image calculated as the average TAC obtained from those voxels in the 70th percentile for the correlation score and, from those, the ones in the $50^{\text {th }}$ percentile for amplitude; this is done to ensure that noisy voxels and voxels are affected by spill-over from surrounding areas are not selected.

Two versions of each study were used: the original one with no prior processing and a masked copy which includes the heart and eliminates the lungs almost completely.

For comparison purposes, manual segmentation was performed on these studies by an experienced biologist. The TACs obtained this way and the ones obtained automatically were compared using the area under the curve:

$$
\varepsilon(\%)=\frac{\sum\left|T A C_{\text {automatic }}-T A C_{\text {manual }}\right|}{\sum T A C_{\text {manual }}} \cdot 100
$$

The algorithm was developed in Java as a pixel-wise plug-in for the PMOD software (PMOD, Zürich, Switzerland). Tests were carried out in an Intel Core 2 Quad CPU Q6600 2.40 $\mathrm{GHz}$ with $4 \mathrm{~GB}$ of RAM.

\section{RESULTS}

The process took a total of $28.9 \pm 1.32$ seconds (mean \pm standard deviation) for the three original ${ }^{13} \mathrm{NH}_{3}$ studies mentioned previously. For the masked version, $36.52 \pm 0.75$ seconds were employed.

In Fig. 2 are displayed the results of the convergence tests for one of the studies. The TAC used for the left ventricle of that same study is shown on Fig. 3 for iterations 1, 2 and 3.

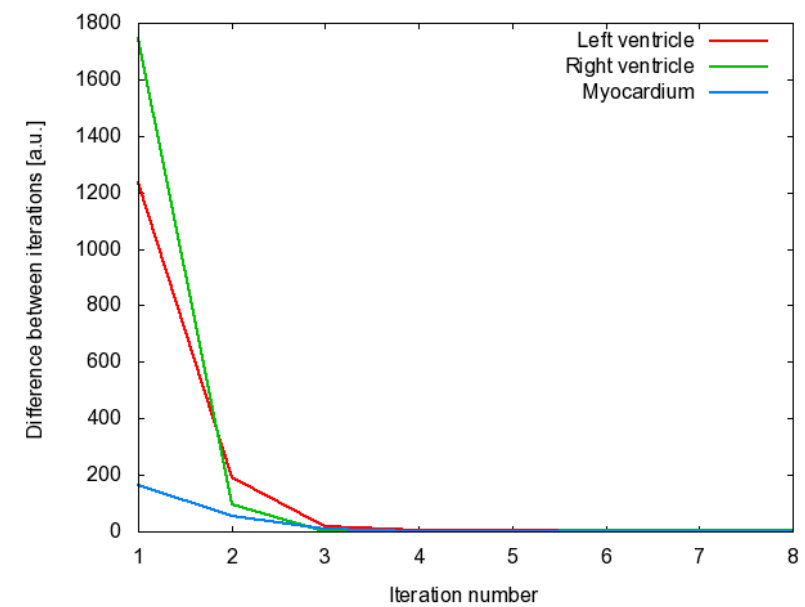

Fig. 2. Differences between the TAC for each region in a given iteration and the one obtained in the next one. After the fourth iteration there is no appreciable difference in either region.

The differences between the automatically obtained TACs and those obtained via manual segmentation can be found on Table I. The correlation between said curves are $r>0.89$ for all cases and specifically $r>0.97$ for the masked cases. 


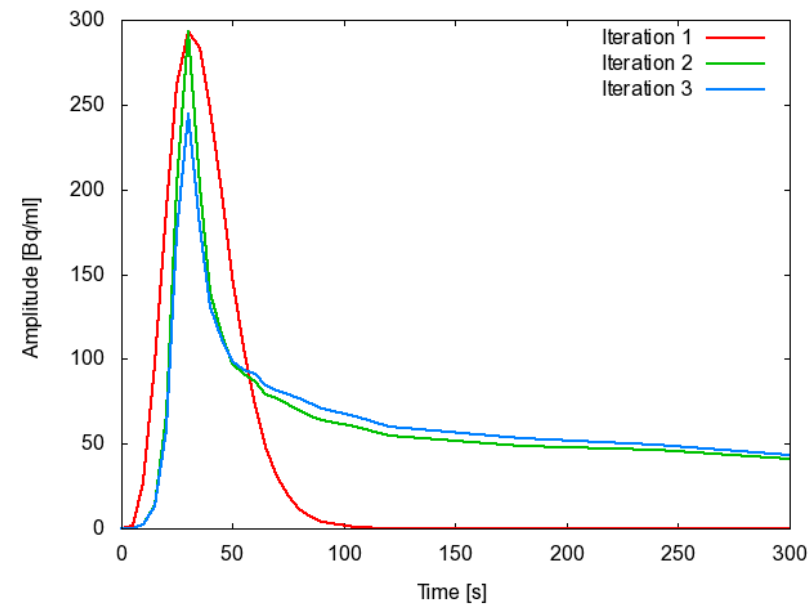

Fig. 3. Evolution of the left ventricle curve in one of the studies tested in this work. From iteration 3 onwards there is no appreciable change. The first curve corresponds to the original analytical curve used to start the iteration. Its maximum amplitude has been levelled to that of the second iteration to perform this comparison. Only the first 300 seconds are displayed.

TABLE I. ERROR RELATIVE TO MANUALLY EXTRACTED TACS (MEAN \pm SD)

\begin{tabular}{llll} 
Preprocessing & Left ventricle & $\begin{array}{l}\text { Rigth } \\
\text { ventricle }\end{array}$ & Myocardium \\
\hline None & $145.91 \% \pm$ & $19.54 \% \pm$ & $13.06 \% \pm$ \\
& $50.18 \%$ & $15.58 \%$ & $10.31 \%$ \\
Masked & $21.82 \% \pm$ & $6.73 \% \pm$ & $9.59 \% \pm$ \\
& $11.8 \%$ & $2.89 \%$ & $2.48 \%$
\end{tabular}

The big differences obtained when the studies are not masked beforehand are mainly due to the presence of the lungs, which TAC is similar to the one from left ventricle (Fig. 4).

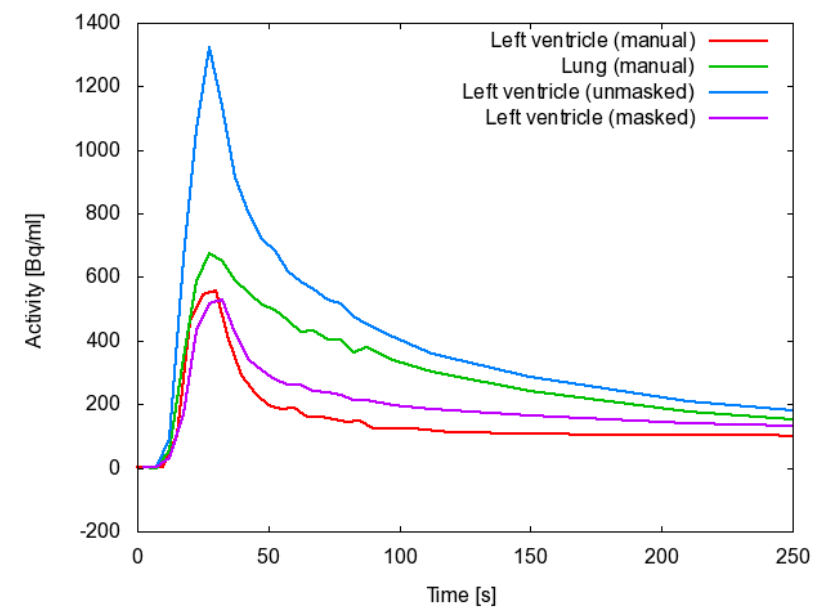

Fig. 4. Differences in extracted TACs for left ventricle in masked and unmasked studies, compared to actual TAC from left ventricle and lung.
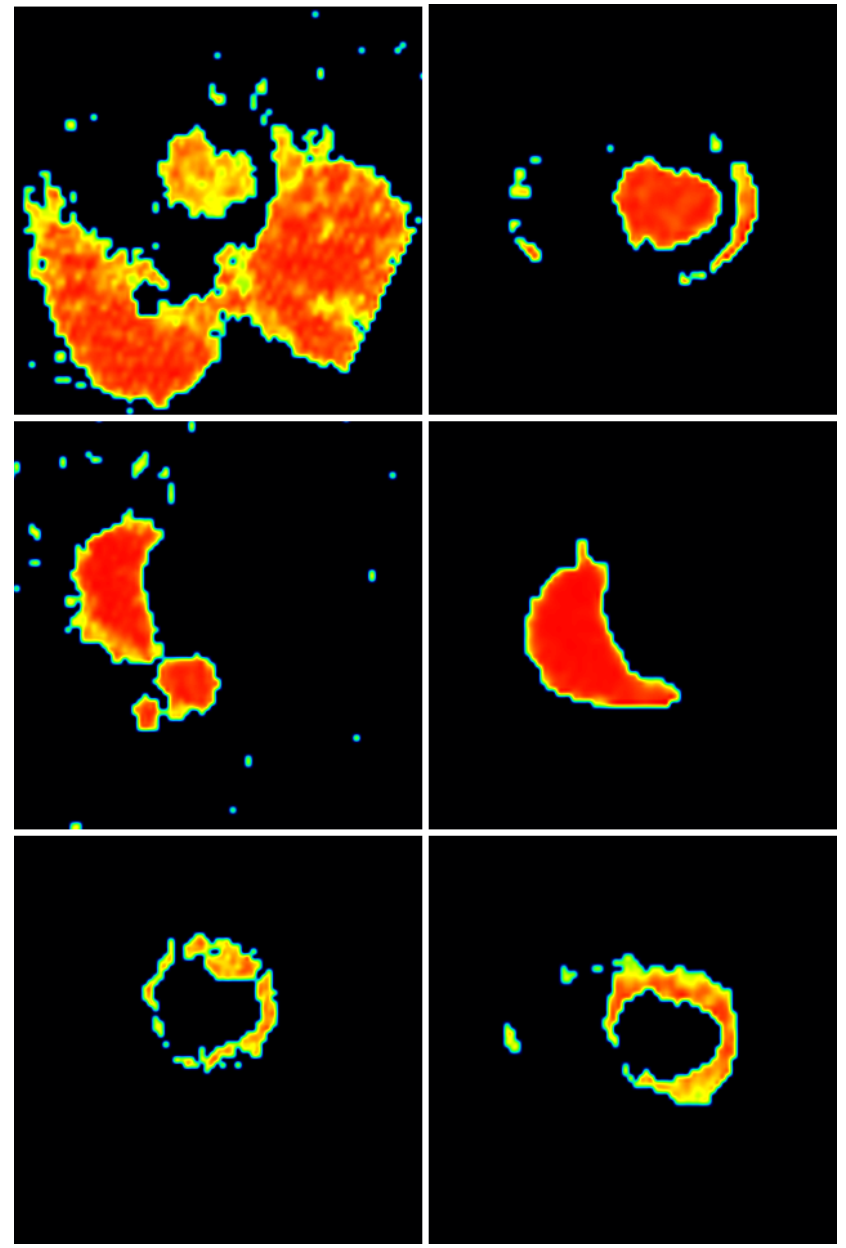

Fig. 5. Correlation images for one of the studies, masked (right) and not masked (left). From top to bottom, displayed regions are left ventricle, right ventricle and myocardium. On the left ventricle image for the masked region, residual lung tissue can still be found.

The regions used for the extraction of each curve are displayed on the main visualization region inside PMOD for verification purposes. An example of said regions is displayed on Fig. 5.

\section{DISCUSSION}

The proposed method was used to successfully extract the TACs from left and right ventricles and myocardium from several swine studies involving ${ }^{13} \mathrm{NH}_{3}$ as a tracer. The calculation of the TACs and the corresponding correlation images took less than 1 minute in the test computer for nine iterations. The results of the convergence test suggest that four iterations could have been used to get approximately the same results. However, the use of a high number of iterations served to test the algorithm stability.

Due to the way the algorithm is defined, there is no spatial overlapping between the correlation images used to extract the TACs from each region, as can be seen in the results (Fig. 5).

When compared to results obtained by directly drawing a ROI over the image, there are bigger divergences in the unmasked studies. This is due to the presence of the lungs, whose TAC mixes with the one belonging to the left ventricle 
(Fig. 4). When the studies are masked as in other previously published works $[7,12,13]$ the error drops, specially in the right ventricle and myocardium TACs. Due to the masking not being optimum (Fig. 5), there is still a small influence from the lungs that causes the left ventricle curve to depart from what is expected.

Masking is therefore obligatory if the TAC extraction is to be done without further post-processing. However, it would also be possible to perform the TAC extraction with no preprocessing and then draw a ROI over the left ventricle in the corresponding correlation image.

The spatial overlapping that represents the biggest shortcoming of factor analysis, as seen for example on [12], is not present at all in the results obtained with this technique; every compartment is strictly separated from each other. This represents an improvement over previous approaches that corrected ambiguous factor analysis solutions [7, 8].

Future work for further development of this techniques include modelling different ways of injection, such as continuous infusion, and more advanced tissue modelling to include later wash-out of the tracer. Also, this iterative correlation approach can be applied to implement blind segmentation techniques.

\section{CONCLUSION}

A new image-derived TAC extraction algorithm has been proposed. Our tests have shown the stability of the results and this method can be used to obtain consistently the imagederived input function from both ventricles. Our implementation is integrated with a widespread research tool (PMOD), thus being very easy to use and evaluate.

\section{REFERENCES}

[1] E. Croteau, E. Lavallee, S. M. Labbe, L. Hubert, F. Pifferi, J. A. Rousseau, S. C. Cunnane, A. C. Carpentier, R. Lecomte, and F. Benard, "Image-derived input function in dynamic human PET/CT: methodology and validation with (11)C-acetate and (18)F-fluorothioheptadecanoic acid in muscle and (18)F-fluorodeoxyglucose in brain," Eur J Nucl Med Mol Imaging, May 2010.

[2] J. E. Mourik, M. Lubberink, A. Schuitemaker, N. Tolboom, B. N. van Berckel, A. A. Lammertsma, and R. Boellaard, "Image-derived input functions for PET brain studies," Eur J Nucl Med Mol Imaging, vol. 36, pp. 463-71, Mar 2009.

[3] M. N. Tantawy and T. E. Peterson, "Simplified [18F]FDG imagederived input function using the left ventricle, liver, and one venous blood sample," Mol Imaging, vol. 9, pp. 76-86, Apr 2010.

[4] A. P. van der Weerdt, L. J. Klein, R. Boellaard, C. A. Visser, F. C. Visser, and A. A. Lammertsma, "Image-derived input functions for determination of MRGlu in cardiac (18)F-FDG PET scans," J Nucl Med, vol. 42, pp. 1622-9, Nov 2001.

[5] D. C. Barber, "The use of principal components in the quantitative analysis of gamma camera dynamic studies," Phys Med Biol, vol. 25, pp. 283-92, Mar 1980.

[6] R. Di Paola, J. P. Bazin, F. Aubry, A. Aurengo, F. Cavailloles, J. Y. Herry, and E. Kahn, "Handling of Dynamic Sequences in Nuclear Medicine," Nuclear Science, IEEE Transactions on, vol. 29, pp. 13101321, 1982.

[7] G. El Fakhri, A. Sitek, B. Guerin, M. F. Kijewski, M. F. Di Carli, and S. C. Moore, "Quantitative dynamic cardiac 82Rb PET using generalized factor and compartment analyses," J Nucl Med, vol. 46, pp. 1264-71, Aug 2005.

[8] A. Sitek, G. T. Gullberg, and R. H. Huesman, "Correction for ambiguous solutions in factor analysis using a penalized least squares objective," IEEE Trans Med Imaging, vol. 21, pp. 216-25, Mar 2002.

[9] A. O. Boudraa, J. Champier, M. Djebali, F. Behloul, and A. Beghdadi, "Analysis of dynamic nuclear cardiac images by covariance function," Computerized Medical Imaging and Graphics, vol. 23, pp. 181-191, 1999.

[10] J. G. Brankov, N. P. Galatsanos, Y. Yongyi, and M. N. Wernick, "Segmentation of dynamic PET or fMRI images based on a similarity metric," Nuclear Science, IEEE Transactions on, vol. 50, pp. 1410-1414, 2003.

[11] M. Magadan-Mendez, A. Kivimaki, and U. Ruotsalainen, "ICA separation of functional components from dynamic cardiac PET data," in Nuclear Science Symposium Conference Record, 2003 IEEE, 2003, pp. 2618-2622 Vol.4.

[12] J. Kim, P. Herrero, T. Sharp, R. Laforest, D. J. Rowland, Y. C. Tai, J. S. Lewis, and M. J. Welch, "Minimally invasive method of determining blood input function from PET images in rodents," J Nucl Med, vol. 47, pp. 330-6, Feb 2006.

[13] H. M. Wu, C. K. Hoh, Y. Choi, H. R. Schelbert, R. A. Hawkins, M. E. Phelps, and S. C. Huang, "Factor analysis for extraction of blood timeactivity curves in dynamic FDG-PET studies," J Nucl Med, vol. 36, pp. 1714-22, Sep 1995. 